\title{
Morphological characterization of Frangula azorica bark as a potential herbal drug
}

\author{
C. Curica* R. Serrano* E. T. Gomes* and O. Silva*
}

* iMed.UL, Faculty of Pharmacy, University of Lisbon, Av. Prof. Gama Pinto, 1649-003 Lisbon, Portugal

odsilva@ff.ul.pt

Frangula azorica V. Grubow (Rhamnaceae), locally known as "sanguinho", is an Azorean endemism inscribed on the IUCN Red List of Threatened Species [1]. Morphological, chemical and biological studies on F. azorica are scarce, but preliminary studies on the bark of this species enable the identification of anthraquinone derivatives as major compounds of this plant part. This kind of compounds are present and responsible for the laxative activity of Frangulae cortex and Rhamni purshianae cortex, two well known herbal drugs of the Western Pharmacopoeias belonging to the same botanical familly (Rhamnaceae) [2].

Comprehensive morphological, chemical and pharmacological studies on F. azorica bark are needed to confirm its potential as laxative, thus bestowing it an interest that may contribute to the preservation of this species.

Hereby we present the results of the application of scanning electron microscopy (SEM) and light microscopy (LM) to the characterization of the $F$. azorica bark. In our study the whole, fragmentised and powdered dried plant material were used. The most useful macroscopic and microscopic characters to identification includes: the channelled external surface of the bark with frequent lenticels and an orange-reddish inner surface; the flattened cork cells with a red content (Fig.1) and a collenchymatous cortex); the mucilaginous glands present in the parenchyma (Fig.2); the groups of sclereids present specially on the cortical parenchyma (Fig.3); the group of phloem fibers with a calcium oxalate prism sheath (Fig.4, Fig.5 and Fig. 7), associated with medullar rays generally bi(Fig.6) and triseriate (Fig.4), and starch grains inside the medullary ray cells (Fig.8).

Identified botanical characters are significant markers of $F$. azorica bark and can, therefore, be used on quality control protocols of this plant part as raw material to pharmaceutical uses.

\section{References}

[1] http://www.iucn.org/ Accessed September (2008).

[2] C. Curica, R. Serrano, E. T. Gomes, O. Silva, Planta Med, 74(2008) 242. 


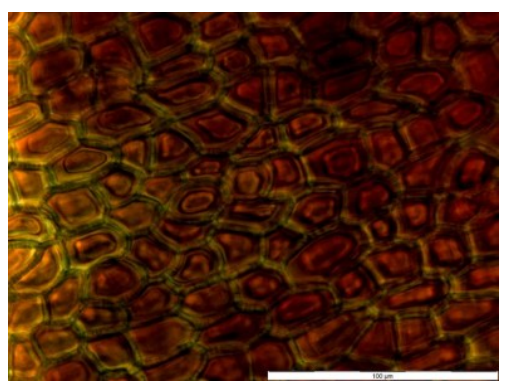

Fig.1. Cork cells. LM surface view detail. Scale bar $=100 \mu \mathrm{m}$.

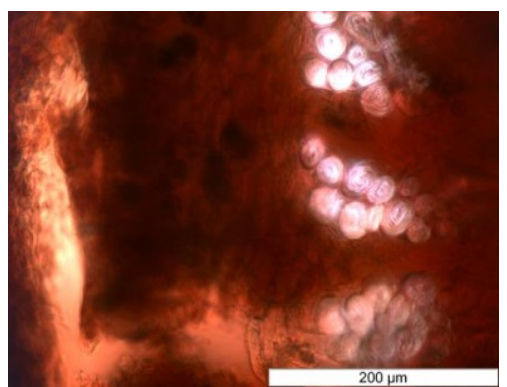

Fig.3. Groups of sclereids on cortical parenchyma. LM transversal section detail. Scale bar $=200 \mu \mathrm{m}$.

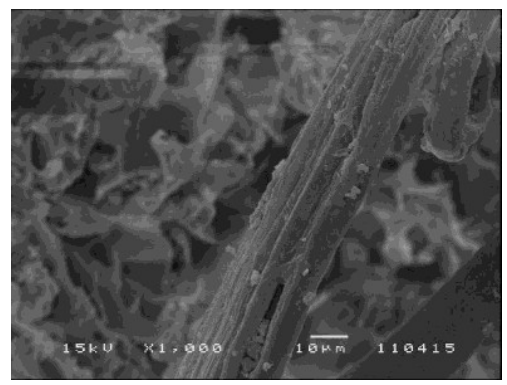

Fig.5. SEM phloem fibers detail. Scale bar $=10 \mu \mathrm{m}$.

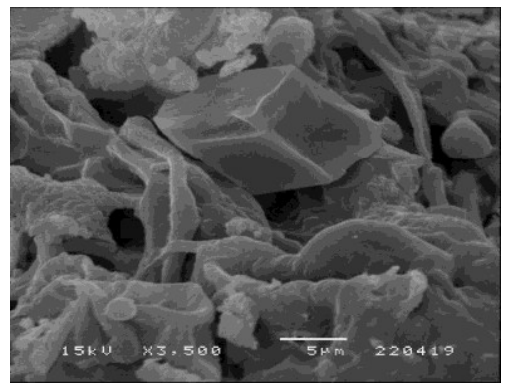

Fig.7. SEM oxalate prism crystal detail. Scale bar $=5 \mu \mathrm{m}$.

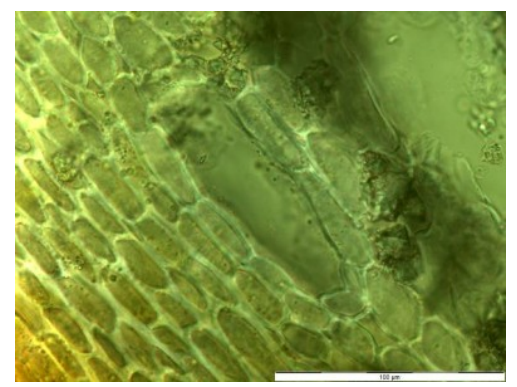

Fig.2. Mucilaginous glands. LM transversal section detail. Scale bar $=100 \mu \mathrm{m}$.

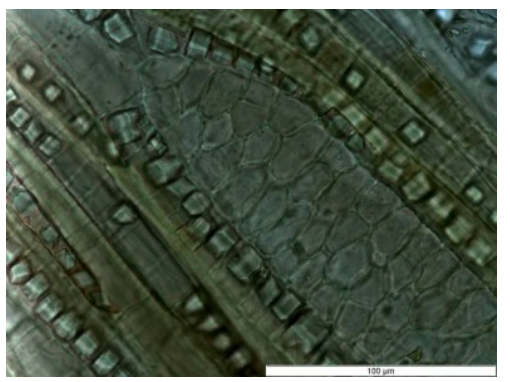

Fig.4. Group of fibers with a calcium oxalate prism sheath, associated with a triseriate medullar ray. LM longitudinal section detail. Scale bar $=100 \mu \mathrm{m}$.

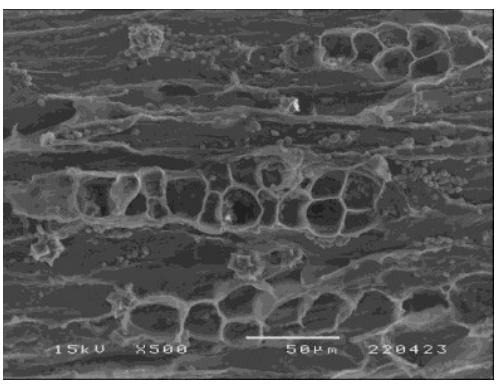

Fig.6. Biseriate medullar rays. SEM longitudinal section detail. Scale bar $=50 \mu \mathrm{m}$.

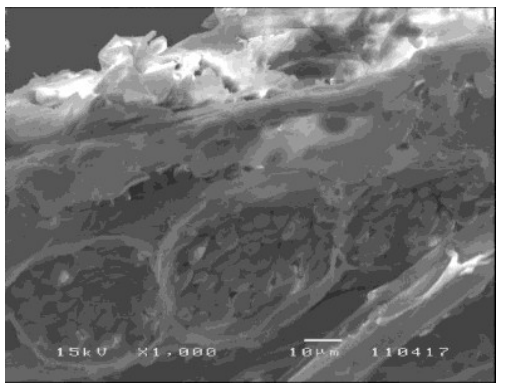

Fig.8. Starch grains inside the medullar ray cells. SEM transversal section detail. Scale bar $=10 \mu \mathrm{m}$. 